\title{
Identification and Classification of Cyberbully Incidents using Bystander Intervention Model
}

\author{
J.I. Sheeba, S. Pradeep Devaneyan, Revathy Cadiravane
}

\begin{abstract}
Cyberharassment is bullying and degrading the adults by means of posting the comments like hurtful and derogatory humor over the internet in an online community. Though few bystanders ever try to reduce the conflicting effects of cyberbullying, and bystanders ever endeavor to interrupt. This will analyze the chattels of articulatory study on bystander intervention using the caricatured procedural made online Social Networking Sites. The proposed works mainly focus on the analysis of direct intervention by bystanders. The direct intervention allows bystanders to do reporting and blocking of cyberbully activities as additional features here. It will generate a report which contains the details of bully by means of alert message and block that bully by the bystander with the victim's permission in the Facebook. This proposed framework will detect cyberbully words from the short hand text and emoticons on the comment sections using Latent semantic analysis (LSA). The Cyberbully words will be classified using a Random Decision Forest algorithm.
\end{abstract}

Index Terms: Bystander Intervention, Cyberbullying Emoticons, Short hand text

\section{INTRODUCTION}

A Social Networking Site (SNS) is online social media platforms which are used by the persons building social networks or social relationship with new community who contribute alike personal or secret information, career activities, history and actual life scenarios. However, SNS provides the space for cyberbullying actions. Nowadays, cyberbullying, which has become gradually common, is a pessimistic circumstance associated with Internet [1]. Cyberbullying or cyber harassment are also known as network (online) bullying. It is a threatening, willful act or behavior that is drifting out by a single or group, using computerized forms of communication, frequency and extra time across a victim who cannot easily oppose themselves. It has been become increasingly common, especially among youths. According to the census of cyberbullying in 2014, 50\% of the youngsters report that they have accomplished repeated bullying through mobile phones or on the internet. Cyberbullying causes a serious threat of substantial and spiritual health to the victims [2].

Bullying is a series of abusive behaviors that are intended and frequently performed task by an individual aiming at insulting or establishing annoyance to others either mentally or

Revised Manuscript Received on July 05, 2019.

J.I. Sheeba, Department of Computer Science and Engineering, Pondicherry Engineering College, Puducherry, India

S. Pradeep Devaneyan, School of Mechanical and Building Sciences, Christ College of Engineering and Technology, Puducherry, India.

Revathy Cadiravane, Department of Computer Science and Engineering, Pondicherry Engineering College, Puducherry, India. physically. These kind of behaviors includes gossips, interpersonal exclusion, spreading rumors, physical or mental attack, and others, that involve a power disproportion between bullies and affected victims (i.e., the victims are afraid of or unable to resist cautiously). The roles of individuals tend to transfer from one group to another group or from one incident to another on the social media. In other words, individuals may have experienced being bullies, victims, or bystanders in cyberbullying incidents and such roles may brunt the possible roles that individuals may assume in future cyberbullying incidents [1]. Numerous laws are established and precautions are taken by parents and teacher to destroy traditional bullying, but when it comes to cyberbullying, the additionally little bit is done. Parents have been seem negligent of cyber bullying happens to their children, as $57 \%$ of who assume their children would come to them when they experience cyberbullying, but in reality only 1 in 3 teens who have been cyber-bullied have told their parents [3]. Bystanders are being in the cyberbullying incidents. Among the teenagers who used SNS, $60 \%$ have witnessed the absolute bullying via that medium. Bystanders in cyberbullying are also called as cyber bystanders can become defenders if they take a positive, active role and, for example, report the bullying incident or relief the victim. They can also take on a negative negative active role and bolster the bullying by reinforcing the perpetration. Alternatively, bystanders can remain as passive if they do not get interacted. By investigating positive bystander behavior, the current research is expected to not only contribute to a complete understanding of teenager's active bystander behavior, but also provide suggestions on how to identify and halt the cyberbullying [2].

To accord stop bullying. Gov, there are various forms of cyberbullying, together with but not restricted to rumor spreading, harassment and posting of humiliating images and words using emoticons (smileys) and short hand text [4].Facebook users more pile of comments and likes. Teenagers are more likely to type cruel things that they would never say to somebody's face. This enables them to post the bullying comments easily. Facebook makes it easy to comment or like on negative posts, allowing numerous children to jump on the bullying position with an easy click of a button. It should be noted that $80 \%$ of students have admitted to detecting recurrent cyberbullying through Facebook. Cyberbullying through Facebook sends the message to the victim post their personal information that the world is beside them and the bullying is deserved. This could result in the sufferer to sense like insulated.

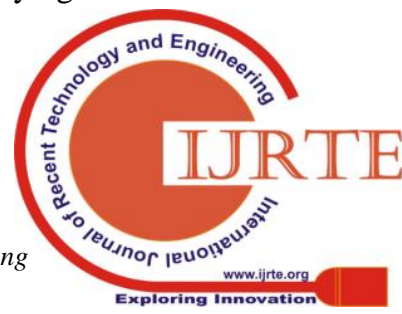


Hence, the proposed work mainly concentrations on the direct intervention by bystanders and identifying the occurrence of cyber bullying activity in online social media platform which helps the government to yield force before many end-users enhancing a target of cyberbullying. This proposed system will give alert messages like sending notification to the cyber-victim and alert message to the bully (another user) in different ways to warn the bully, identifying short hand text and emoticons on the comment sections. This also generating a report which contains the details of bully and also block that bully by the bystander with victim's permission. The cyberbully words will be classified using RDF algorithm. It will help to protect the user from an unhealthy environment. Finally, this work will give the connotation for accepting bystander effect in cyberbullying, and how to authorize scheme solutions to raise your spirits bystander intervention in online social media platform.

\section{RELATED WORKS}

Cheng-Yu Lai, et al., the application examines the relationship among the individual's networked disinhibiting behavior and their interest of excitement while using cyberbullying. However, networked disinhibiting can be used to moderately describe the reasons, the interrelations among individual's motivation, unstate condition, and benefit of automation and cyberbullying behavior remain topics that needs advance inquiry [1].Samuel Owusu, et al., this research understands whether teenager's empathy has an impact on their positive bystander behavior and proposed two hypotheses about the effect of empathy due to harassment. The study has to increase sample size, improving the reliability of the affective empathy scale, and investigating possible moderating factors [2].Ziyi Li, et al., an automated cyberbullying identification method that accomplish the parent-child interrelationship among comments in the post to arrest the attitude from the victim to identify cyberbullying comments [3].Arpita Chakraborty, et al., provided a design model that possess a superior semantic understanding of cyberbullying messages and can identify different types of bullying that can occur with/without the presence of profane/abusive words. But it cannot detect the difference profound signals present in bullying messages and responses, i.e., emotional will help in accurately identifying different forms of bullying and foster a deeper understanding of their impact on recipients [4]. Finally, complete content and organizational editing before formatting. Please take note of the following items when proofreading spelling and grammar. Dominic Di Franzo, et al., bystander intervention intimate the false effects of bullying and they accepted various kinds of data around the audience size and viewing notifications designed to enhance a sense of particular authority in bystanders. Although most of the bystanders could not intervene directly, they simply generate reports and send to the Victim [5].Peijun Zhao, et al., examined the correlation between emoticon usages and tweet comfortable, tweet architecture, user enumeration using an mmGRU model for predicting emoticon categories and positions motivated by the observations. This system could not manipulate a list of case histories in display and various types of emoticons are used in social media [6].Chenliang $\mathrm{Li}$, et al., promoted the

linguistically associated words beneath the similar category when the fragmented process, by using the Generalized Polya Urn (GPU) model, enlarged the types same for two linguistically associated words that may not occur many times in the short hand text selection. This system does not concentrate more on topic inference strategy and less accuracy in detecting computer short form of words [7].

Mohammadreza Rezvan, et al., contributed both a character comments collection and an abusive word dictionary takes various categories of stalking contented such as, intellectual, appearance - related harassment, political, racial and sexual. The system could not perform analysis on harasser and victim's language [8].Santosh Joshi, et al., construct a machine learning technique for malware identification using Random Forest classifier for the action series information taken from the virtual machine surroundings. It does not include the implementation of additional classification algorithms and performing a comparison to identify the best algorithm for analyzing the process list data structures [9]. Jacob L. Bigelow, et al., elaborates initial conclusions for an application that uses Latent Semantic Indexing (LSI) for the cyberbullying detection in an acknowledged selection of posts from Formspring. Me. This system does not test in other domains, like a collection of SMS messages or Twitter and Facebook posts [10].

Xiang Zhi Tan, et al., explored an efficient approach for robots reduce human being offense: activating bystander interventions. The disadvantages of this application are, need to achieve without permanent guidance in personal surroundings [11]. Lindsay Blackwell, et al., this approach explains the architecture and prediction of HeartMob, a platform constructed by and for the people who are extremely concerned with the most severe online forms of impersonation. Moreover, the platform guidelines and broadcasting apparatus are created for the apparently similar user base and do not explanation for respective one's involvement and applications of social brutality [12]. Jessica Vitak, et al., displayed the ramification of aspect correlate with women's online cyberbullying and point to the demand for both philosophers and engineers to continue their efforts to decrease cyberbllying behaviors and reduce their pessimistic effects. This does not extend the most empathetic of the present state of online annoyance for women and unable to construct more efficient apparatus based on what we already know more about the adults factors combined with intimidation in social media [13].

Noviantho, et al., introduced to build a design for classification with excellent certainty in detecting cyberbully comments using the Support Vector Machine (SVM) and Naive Baye's method. The classification of cyber bullying on text conversations that also contains shorted hand text is not detected. Furthermore, organizing text in the comments are further, denouncing due to text in the comments frequently have shorted hand texts [14].Rahat Ibn Rafiq, et al., this proposed system 
introduced an eminently extensible without signifying veracity and deeply responsible for establishing signals. This system does not develop a diverse, scalable and responsive classifier with better precision and recall performances for Vine as well other diverse SNS like Facebook [15]. Vivek K. Singh, et al., this system introduces the usage of computerized text and image analysis Application Programming Interface for the multi-modal (textual and visual) cyberbullying identification and descriptions that the achievements of computerized cyber bullying detector enhances with the usage of a multi-modal visual + textual) technique when correlated to a text only technique. In addition, research efforts could leverage the availability of emoticon contents as well as short words for better cyber bullying detection [16]. Elaheh Raisi and Bert Huang aim to improve automated detection of cyber bullying presently advancing weakly supervised techniques that are furthermore evaluate the online countenance or the progression of comments in the post. Although, the detection of an automated cyberbully is the main defect between lots that must be clarified to competently addressed the cyberbullying anomaly [17]. MauroColetto, et al., investigated the end-users who can accept abusive language and antagonism words in Facebook and Twitter and other social media by analyzing their profile pictures. Moreover, aggressive users smile less, and they appear not happy in their profile pictures, dominated by fear, sadness and anger using these particulars the aggressive users cannot able to detect, so it provides less accuracy [18].

Based on the above Literature Survey to overcome the drawbacks of the various data around the audience size and viewing notifications at the time of cyberbullying [5], this proposed system is going to identify a cyberbully word from emoticons and short hand text in the comments section and categorized such as Denigration, Trickery, Flaming and Cyberstalking which helps the society to forbid the cyberbullying victims from facing the troubles of cyberbullying.

\section{Proposed System}

In this proposed architecture shown in Figure 1, the mechanism of identifying cyberbully action starts from an input absorption is taken from the SNS. Input are like comments which have frequently occurred in the post collected from Facebook. Inputs are given to the data preprocessing steps that are tested to advance the trait of an information to be researched, successive diagnostic steps that includes tokenization, removing special characters, stop words, stemming and coreference resolution.

After doing preprocessing of the absorption data, the output will be given to the Feature Extraction. It is performed to obtain elements like pronoun, adjective, noun, short hand text in the comments, statistics on the presence of the words in the posts. Using the training dataset the preprocessed online social media comments will be tested for bullying word presence using LSA technique in natural language processing, that may be due to the occurrence of term weighting scheme, it will apply to the posts, astounding a LSA and detect the cyberbully words. For cyberbully Classification such as Flaming, Cyber Stalking, Denigration and Trickery, Random
Forest Classifier is used. A Random Forest classifier is very easy to measure the dependent importance of each element on the prognosis.

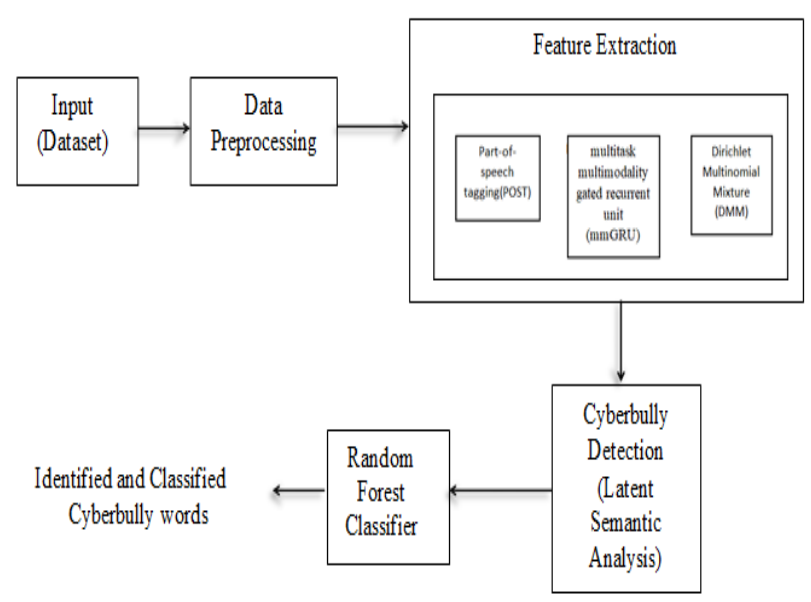

Figure 1.Proposed Framerrork of Cyberbully Detection and Classification system

This proposed architecture for cyberbully detection activities, some specific actions has been followed

- Input

- Data Preprocessing

- Feature Extraction

- Cyberbully detection

- Random Forest Classifier

\section{A. Input}

Comments which have frequently occurred in the post collected from social networks (Facebook) are given as input. The datasets include the comments that are posted by users, images, video clips on networking sites, nearly 100 comments will be taken as the input to the proposed system.

\section{B. Data Preprocessing}

The data preprocessing is used for representing the data in character area to the classifiers. Preprocessing of data where the dataset is processed so that data contains only required information. Subsequently, de-noised words are analyzed. The removal of white spaces and stop words can be considered as a way of data preprocessing after which tokenization and lemmatization occurs.

\section{Feature Extraction}

It is used for extracting the data, short hand text and emoticons required for the processed input. Parsing pronoun, noun and adjective associates two steps they are part-of-speech tagging (POST tagging) and extracting pronoun, noun and adjective from the identified output and data on the presence of a word in the comments among the posts is also extracted. A multitask multimodality Gated Recurrent Unit (mmGRU) is used to identify the positions and types of emoticons and to tentatively define the interrelationships between emoticons and Facebook theme, Facebook design, user demographics. This framework helps averaging the strong interrelationships 
between emoticon categories and their positions for enhancing the performance of both tasks [6]. Dirichlet Multinomial Mixture (DMM) model is used to competent for the categorical assumption over the short texts and domineering this every piece of short text into the single category which is produced for more categories Poisson Dirichlet Multinomial Mixture (PDMM) is used. This model is named DMM and also called as PDMM [7].

\section{Cyberbully detection}

The Cyberbully detection is accomplished by using the LSA technique in natural language processing, on appropriate circulation linguistics, by calculating interrelationships among a set of posts in the comments and the conditions they accommodate by generating the set of concepts associated to that posts or conditions [7]. It is used for analyzing, if the application will be able to identify the latent semantics, even with a very shortened post, that could not obtain all of the cyberbully words, short hand text and emoticons. To detect the cyberbullying in short hand text posts that are scattered with abbreviations, unusual punctuation, misspellings and cyberbully words, short hand texts and emoticons. The application is not based on a database of bullying terms and conditions, and, then, awaits on LSI for semantic analysis.

\section{E. Random Forest Classifier}

After cyberbully words, short hand text and emoticons are detected, these words are classified using random forest algorithm. RDF is very easy to measure the corresponding attention of every feature on the prediction and works efficiently when compare to traditional classification algorithm [9]. It gives an assessment value of what variables are important in the classification. In this proposed system, it will classify the cyberbully words into four categories: Flaming, Denigration, Stalking and Trickery.

- Flaming is described as hateful comments occurred in the posts (for example, insulting, name-calling, swearing or otherwise posting in an abusive language in the comments section) over other users (personal attacks) or her or his thoughts in electronic transmission. Flaming was mostly seen on controversy sites (open groups or sites on Facebook). Moreover, it was found that Facebook users have a pessimistic attitude against flaming on Facebook.

- Denigration is the online spread of gossip and other abusive material to harm the influence or friendships and send or publish rumors on the Internet modified images of the victim to harass them. It is also called as "chit chat", The main goal of Denigration is to depress the target adult.

- Cyberstalking causes an extreme distress for the victim, by spreading false rumors. It can impact their career, personal relationships, and aspect of life. Often time victims do not know who is the perpetrator and start wondering if they are being watched or followed.

- Trickery or Outing is an intentional act of confusing the adults through the posting of sensitive, private or embarrassing information without the victim's permission in the comments among the social media [8].

After cyberbully word extraction, these words are given to the random forest classifier. This algorithm will classify the words based on their categories. The input from a cyberbully word extraction with features, elements and labels into a decision tree, it will formulate a few sets of the decree, which will be used to make the prognosis. It can handle thousands of text variables without variable deletion. Based on this working principle the RDF will be classifying the cyberbully words.

\section{Performance Metrics}

The metrics to be considered for the proposed work will be deliberated using several quality measurement techniques, namely Precision, Recall, F-Measure, Classification accuracy, RMSE, Specificity and Sensitivity.

\section{A. Precision}

Precision is the fraction of fetched Cyberbully words, emoticons and short hand texts that are consistent with the find.

Precision $=\{$ No. Of Related short hand texts, emoticons and Cyberbully words $\} \cap\{$ No. Of Rescued short hand texts, emoticons and Cyberbully words $\} /\{$ No. Of Rescued short hand texts, emoticons and Cyberbully words $\}$

\section{B. Recall}

Recall is the fraction of the cyberbully words, emoticons and short hand texts that are consistent to the queries that are successfully rescued.

Recall $=\{$ No. Of Related short hand texts, emoticons and Cyberbully words $\} \cap\{$ No.Of Rescued short hand texts, emoticons and Cyberbully words $\} /$ \{No. Of Related short hand texts, emoticons and Cyberbully words \}

\section{F-Measure}

F-Measure computes both Recall and Precision. It can be predicted by using the given equation.

$\mathrm{F}=2$. precision. recall/precision+recall

\section{Classification Accuracy}

Classification Accuracy determines the percentage of correctly analyzed cyberbully words, emoticons and short hand texts and it is predicted by using the following equation. Accuracy $=(\mathrm{TP}+\mathrm{TN}) /(\mathrm{TP}+\mathrm{TN}+\mathrm{FP}+\mathrm{FN})$

The aspect of cyberbully identification, the terms are determined in the following manner: TP- Determined as a word, emoticons and short hand texts being classified correctly as relating to a cyberbully category.

TN-Determined the words, 
emoticons and short hand texts which were non-cyberbully words

FP-Determined as a cyberbully word, emoticons and short hand texts, even if it is in the non-cyberbully category

FN-Determined as a non-cyberbully word even if it is in superbly category

\section{E. Root Mean Square Error (RMSE)}

It is the variance between identifying and classifying the cyberbully words, emoticons and short hand texts estimated by a system and the cyberbully words, emoticons and short hand texts actually observed from the input. It is predicted by using the following equation.

$$
\text { RMSE }=\sqrt{\frac{\sum_{i=1}^{n}\left(X_{o b s, i}-X_{\bmod 1, i}\right)^{2}}{n}}
$$

Where, $X_{\text {obs }}$ is manually classified cyberbully words, emoticons and short hand texts and $\mathrm{X}$ model are system classified cyberbully words, emoticons and short hand texts a time/place $\mathrm{i}$. $\mathrm{N}$ is the number of inputs.

\section{F. Sensitivity (also called True Positive Rate)}

Sensitivity has the ability to detect the condition correctly. It will classify the cyberbully words, emoticons and short hand texts which are under the cyberbully words category in the given input. It is predicted by using the following equation.

Sensitivity $=\mathrm{TP} /(\mathrm{TP}+\mathrm{FN})$

\section{G. Specificity (also called True Negative Rate)}

Specificity has the ability to eliminate a condition correctly. It will classify cyberbully words, emoticons and short hand texts which are not under the cyberbully words category in the given input. It is predicted by using the following equation.

Specificity $=(\mathrm{TN}+\mathrm{FP})$

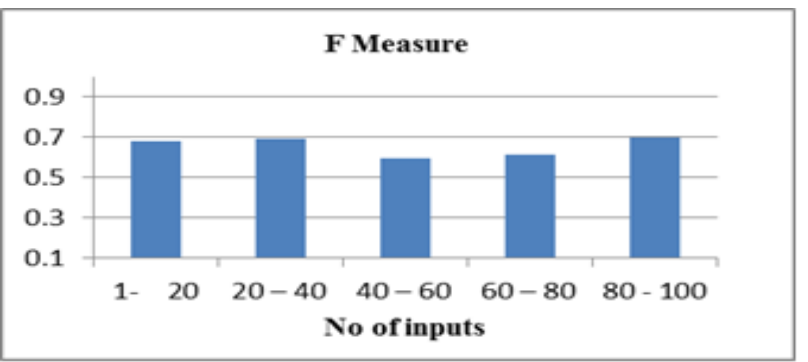

Figure 2: F- Measure

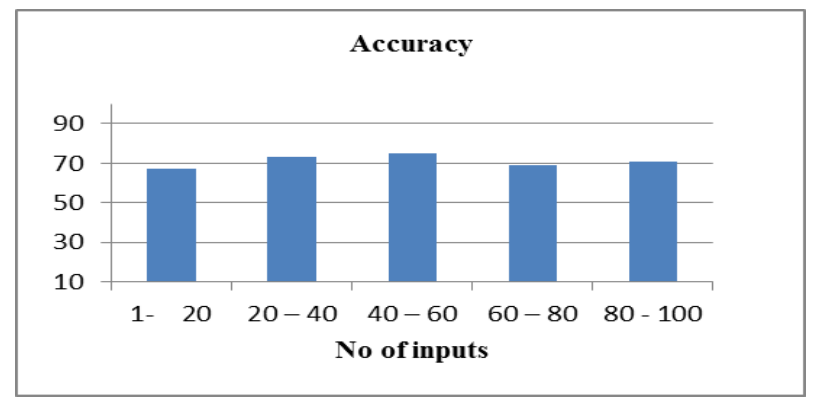

Figure 3: Classification Accuracy

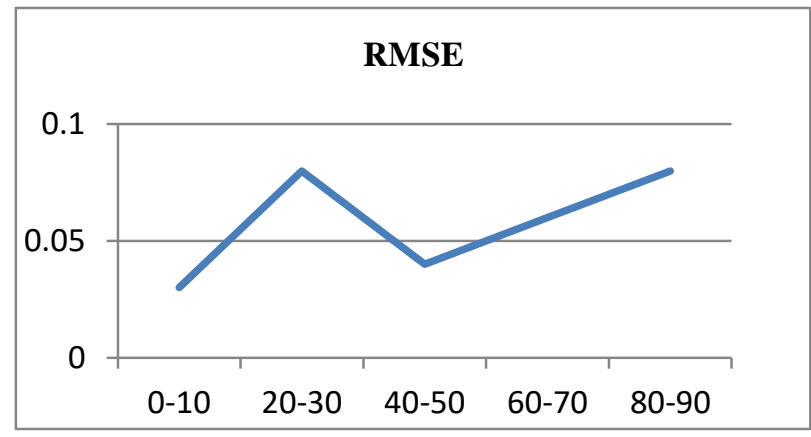

Figure 4: RMSE Graph

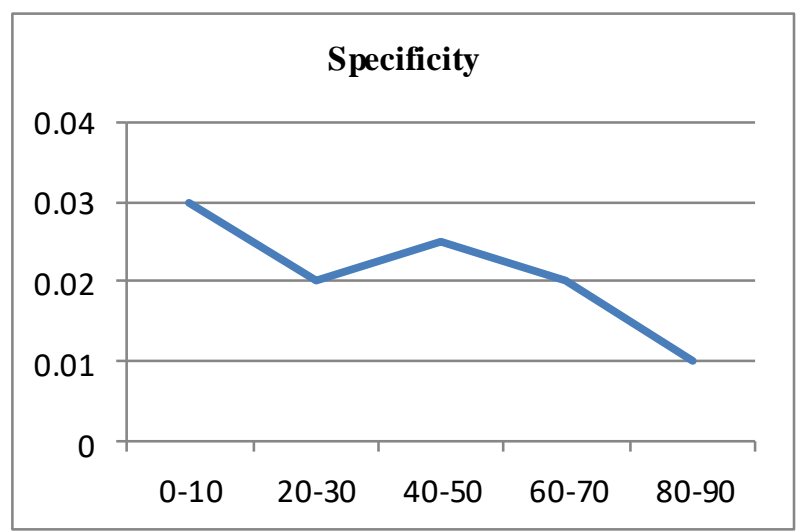

Figure 5: Performance analysis based on Specificity Values

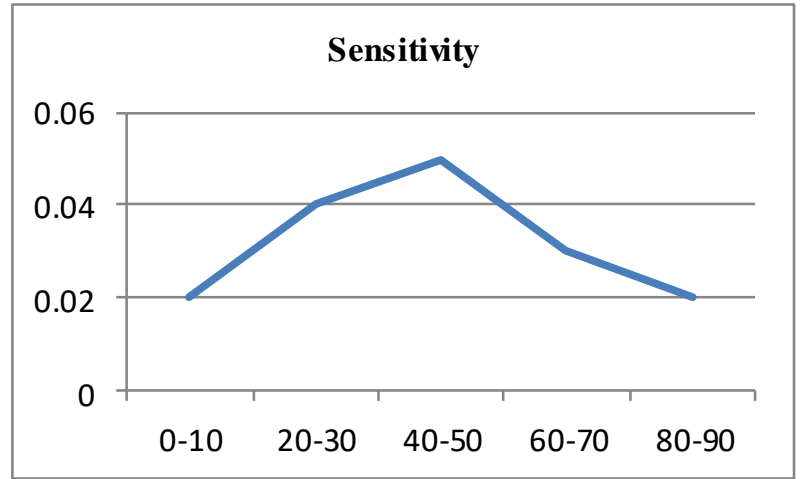

Figure 6: Performance analysis based on Sensitivity Values

Figure 2 shows the F-measure obtained with different values. The performance of the system is evaluated with posts collected from Facebook. It is observed from Figure 2 which shows the F-measure values obtained. Figure 3 shows the classification accuracy graph obtained using different values. Figure 4 shows the RMSE graph. Sensitivity and specificity values are calculated by randomly collected 50 comments, and the results obtained using the given data set are shown in Figures 5 and 6.

\section{Conclusion}

Cyberbullying is a frequently occurring social problem that is happening on the Internet. The internet is convenient environmental surrounded for bullying the most vulnerable community.

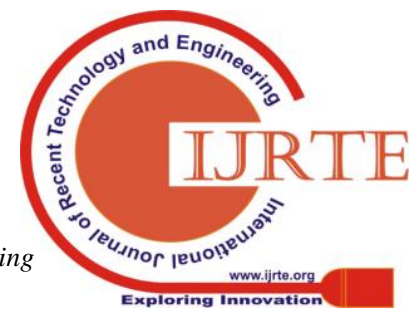


Machine Learning representation and authentication make automatic revelation of bullies' messages in online media possible and ensures building a relevant and clear social media environment. This proposed system mainly concentrated on identifying the presence of cyber bullying activities in the social media platform using Latent Semantic Analysis, multitask multimodality Gated Recurrent Unit and Dirichlet Multinomial Mixture which helps many end-users enhancing a sufferer of cyberbullying. This system also uses the Random Forest Classifier which helps to identify the cyberbullying actions and categorized it as Denigration, Trickery, Flaming and Cyberstalking. The productive visualization can be promisingly met with the help of simpler notification about the occurrences of bullies. After identifying the cyberbullying problem, relevant measures should be taken to prohibit further molestation of victim, preventing the spread of vulgar and immense gossips among the social media. Analyzing, verifying and providing additional information helps the victims to take measures for getting rid of the problem.

\section{REFERENCES}

1. Cheng-Yu Lai and Chia-Hua Tsai, "Cyberbullying in the Social Networking Sites: An Online Disinhibition Effect Perspective", Third Multidisciplinary International Social Networks Conference on Social Informatics, 2016, pp.1-4.

2. Samuel Owusu and Lina Zhou, "Positive Bystanding Behavior in Cyberbullying: The impact of empathy on adolescents' cyber bullied support behavior", IEEE Conference on cybersecurity, 2015, pp.163-165.

3. Ziyi Li, Junpei Kawamoto, Yaokai Feng and Kouichi Sakurai, Cyberbullying Detection Using Parent-Child Relationship between Comments", 18th International Conference on Information Integration and Web Based Applications and Services, 2016, pp.325-334.

4. Arpita Chakraborty, Yue Zhang and Arti Ramesh, "Understanding Types of Cyberbullying in an Anonymous Messaging Application", WWW'18 Companion Proceedings on The Web Conference, 2018, pp.1001-1005.

5. Dominic DiFranzo, Samuel Hardman Taylor, Franccesca Kazerooni, Olivia D Wherry and Natalya N Bazarova, "Upstanding by Design: Bystander Intervention in Cyberbullying", CHI Conference on Human Factors in Computing Systems, 2018 pp.1-12.

6. Peijun Zhao, Jia Jia, Yongsheng An, Jie Liang, Lexing Xie and Jiebo Luo, "Analyzing and Predicting Emoji Usages in Social Media", WWW'18 Companion Proceedings on The Web Conference, 2018, pp.327-334.

7. Chenliang Li, Yu Duan, Haoran Wang, Zhiqian Zhang, Aixin Sun and Zongyang Ma, "Enhancing Topic Modeling for Short Texts with Auxiliary Word Embeddings", ACM Transaction on Information Systems (TOIS), Vol no. 36(2), 2017, pp.11:1-11:30.

8. Mohammadreza Rezvan, Saeedeh Shekarpour, Lakshika Balasuriya, Krishnaprasad Thirunarayan, Valerie L. Shalin and Amit Sheth, "A Quality Type-aware Annotated Corpus and Lexicon for Harassment Research", 10th ACM Conference on Web Science - WebSci'18, 2018, pp.33-36.

9. Santosh Joshi, Himanshu Upadhyay, Leonel Lagos, Naga Suryamitra Akkipeddi and Valerie Guerra, "Machine Learing Approach for Malware Detection Using Random Forest Classifier on Process List Data Structure", 2nd International Conference on Information System And Data Mining, 2018, pp.98-102.

10. Jacob L. Bigelow, April Edwards and Lynne Edwards, "Detecting Cyberbullying using Latent Semantic Indexing”, First International Workshop on Computational Methods for CyberSafety, 2016, pp.11-14.

11. Xiang Zhi Tan, Marynel Vazquez, Elizabeth J, Carter, Cecilia G. Morales and Aaron Steinfeld, "Inducing Bystander Interventions During Robot Abuse with Social Mechanisms", ACM/IEEE International Conference on Human-Robot Interaction, 2018, pp.169-177.
12. Lindsay Blackwell, Jill Dimond, Sarita Schoenebeck and Cliff Lampe, "Classification and Its Consequences for Online Harassment", ACM on Human-Computer Interaction, Vol. 1, 2017, pp.24-1.

13. Jessica Vitak, Kalyani Chadha, Linda Steiner and Zahra Ashktorab,"Identifying Women's Experiences With and Strategies for Mitigating Negative Effects of Online Harassment”, ACM Conference on Computer Supported Cooperative Work and social Computing, 2017, pp.1231-1245.

14. Noviantho, Sani Muhamad Isa and Livia Ashianti, "Cyberbullying Classification using Text Mining", 1st International Conference on Informatics and Computational Sciences, 2017, pp.241-246.

15. Rahat Ibn Rafiq, Homa Hosseinmardi, Richard Han, Qin Lv and Shivakant Mishra, "Scalable and timely detection of cyberbullying in online social netwoks", 33rd Annual ACM Conference/Symposium on Applied Computing, 2018, pp.1738-1747.

16. Vivek K. Singh, Souvick Ghosh and Christin Jose, "Towards Multimodal Cyberbullying Detection”, CHI Conference Extended Abstracts on Human Factors in Computing Systems, 2017, pp.2090-2099.

17. Elaheh Raisi and Bert Huang, "Cyberbullying Detection with Weakly Supervised Machine Learning”, IEEE/ACM International Conference on Advances in Social Networks Analysis and Mining, 2017, pp.409-416.

18. Mauro Coletto, Claudio Lucchese and Salvatore Orlando, "Do Violent People Smile: Social Media Analysis of their Profile Pictures", WWW'18 Companion Proceedings on The Web Conference, 2018, pp.1465-1468.

\section{AUTHORS PROFILE}

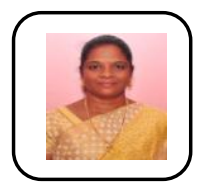

J.I. Sheeba has completed her B.E, M.E in Computer Science and Engineering. She has complted her Ph.D in Text mining area from Pondicherry University. Presently she is working as an Assistant Professor in Department of Computer Science and Engineering, Pondicherry Engineering College and has more than 15 years teaching experience. She has published papers in many international journals and conferences. Her research interest includes Data mining, soft computing and Information Security.

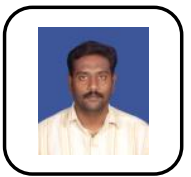

S. Pradeep Devaneyan has completed his B.E Mechanical Engineering (Karunya Institute of Technology), M.E CAD/ CAM (Arulmigu Kalasalingam College of Enginering and Technology) and Ph.D from Pondicherry University. At present working as Professor in Christ College of Engineering and Technology, Puducherry and has 15 years of teaching experience. He has published many papers in reputed journals and conferences.

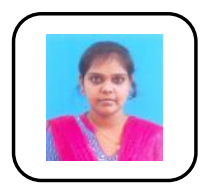

Revathy Cadiravane her BTech in Computer Science and Engineering from Pondicherry University and MTech in Information Security from the Pondicherry Engineering College.. Her areas of interest are text Mining, Information Security and data analytics. 